\title{
Efeitos da semente de sucupira-branca (Pterodon emarginatus, Vogel) sobre a inflamação na articulação coxofemoral em cães avaliados pela termografia
}

\author{
[Effects of white sucupira (Pterodon emarginatus, Vogel) seed on inflammation of the coxofemoral \\ joint in dogs evaluated by thermography]
}

\section{"Artigo Científico/Scientific Article"}

\author{
Fernanda Lúcia Passos Fukahori ${ }^{1}$, Michelle Suassuna de Azevedo Rêgo ${ }^{1}$, Vanessa Carla Lima Silva ${ }^{2}$, \\ Daniela Maria Bastos Souza ${ }^{3}$, George Chaves Jimenez ${ }^{3}$, Evilda Rodrigues de Lima ${ }^{1 *}$
}

\author{
${ }^{1}$ Departamento de Medicina Veterinária, Universidade Federal Rural de Pernambuco, Recife-PE, Brasil. \\ ${ }^{2}$ Médica Veterinária autônoma, Recife-PE, Brasil \\ ${ }^{3}$ Departamento de Morfologia e Fisiologia Animal, Universidade Federal Rural de Pernambuco, Recife-PE, Brasil. \\ *Autora para correspondência/Corresponding author: E-mail: evilda17@ hotmail.com
}

\begin{abstract}
Resumo
Objetivou-se verificar o efeito anti-inflamatório na articulação coxofemoral em cães, do extrato da semente de sucupira-branca utilizando como veículo o Biotônico Fontoura ${ }^{\circledR}$. Obteve-se o extrato por meio da associação com o Biotônico Fontoura ${ }^{\circledR}$, que permaneceu sob maceração por sete dias em refrigeração a $4^{\circ} \mathrm{C}$, sendo posteriormente filtrado e acondicionado em frasco âmbar, fechado, devidamente identificado. Obteve-se o Meloxicam no comércio local. Após a anamnese, os exames complementares e a avaliação termográfica, 23 animais que se encaixavam nos pré-requisitos receberam todas as instruções. Os animais do grupo fito receberam um frasco com o extrato, para ser administrado $2,5 \mathrm{~mL}$ na dose e $300 \mathrm{mg} / \mathrm{kg}$, via oral, diariamente pela manhã, por 21 dias; os do grupo alopático receberam 21 comprimidos de Meloxicam, para ser administrado conforme o fabricante. Os animais foram acompanhados pela termografia nos tempos $\mathrm{T}=0$ dias, $\mathrm{T}=10$ dias e $\mathrm{T}=21$ dias, sendo avaliados hematológica e radiograficamente no início e no fim da pesquisa. Animais do grupo fito apresentaram efeito positivo do extrato na resolução do processo inflamatório da articulação, sendo $61 \%$ mais eficaz do que o grupo Meloxicam. Não houve alterações nos parâmetros hematológicos, bioquímicos e radiográficos.
\end{abstract}

Palavras-chave: artrite; Biotônico Fontoura ${ }^{\circledR}$; Meloxicam; termodiagnóstico; temperatura.

\begin{abstract}
The aim of this study was to verify the anti-inflammatory effect in the joint of dogs using sucupira seed extract and Biotônico Fontoura ${ }^{\circledR}$ as a vehicle. The extract was obtained from the association with the Biotônico Fontoura ${ }^{\circledR}$, which remained under maceration for seven days at $4^{\circ} \mathrm{C}$, after which it was filtered and stored in a closed, properly identified, amber bottle. Meloxicam was obtained from local commerce. After anamnesis, complementary examinations and thermographic evaluation, 23 animals that fit the prerequisites received instructions for use. The animals in the phyto group received a vial of the extract to be administered $2.5 \mathrm{~mL}$ per dose and $300 \mathrm{mg} / \mathrm{kg}$ orally daily for 21 days and those in the allopathic group received 21 tablets of Meloxicam to be administered as described by the Manufacturer. Animals were followed by thermography at times $T=0, T=10, T=21$, being evaluated hematologically and radiographically at the beginning and at the end of the study. The animals of the phyto group showed a positive effect of the extract in the resolution of the inflammatory process of the joint, being $61 \%$ more effective than the Meloxicam group. There were no changes in hematological, biochemical and radiographic parameters.
\end{abstract}

Keywords: arthritis; Biotônico Fontoura ${ }^{\circ}$; Meloxicam; thermodiagnosis; temperature.

\section{Introdução}

Dentre vários tratamentos empíricos à base de plantas, estão os que utilizam a sucupira-branca

(Pterodon emarginatus Vogel), que na medicina popular é conhecida como faveiro, sucupira- 
branca, fava-de-sucupira, sucupira ou sucupira-lisa (Lorenzi e Matos, 2002). Sinais de neurotoxicidade foram exibidos por alguns animais durante a fase experimental, porém esse fitoterápico foi proporcional à concentração do extrato, não sendo letais na dose de $2.000 \mathrm{mg} / \mathrm{kg}$ por via oral (Fukahori et al., 2015).

Os principais constituintes ativos responsáveis pelas propriedades curativas das plantas advêm do seu metabolismo secundário e são gerados por meio de biossíntese de estruturas complexas como alcaloides, terpenoides e alguns diterpenos que estão diretamente envolvidos na inibição de mediadores da inflamação (Spindola et al., 2010; Moraes et al., 2012). O efeito antiinflamatório dos flavonoides encontrados na sucupira-branca (Pterodon emarginatus, Vogel) é explicado pelo seu efeito inibitório sobre o metabolismo do ácido araquidônico (O'Reilly et al., 2000).

O termo osteoartrite se origina de uma etiologia inflamatória podendo estar associada às causas secundárias relacionadas com $\mathrm{o}$ envelhecimento, trauma, lesões crônicas, inflamação local, obesidade, displasia da articulação coxofemoral entre outros fatores. Os sinais clínicos, muitas vezes, não são compatíveis com os achados radiográficos, observando-se desde um pequeno desconforto até dor intensa ao toque, claudicação e paresia voluntária dos membros posteriores podendo evoluir para a doença articular degenerativa (Nogueira e Tudury, 2002; Nogueira et al., 2005; Rocha et al., 2013).

$O$ diagnóstico na fase precoce da osteoartrite, entretanto, é especialmente difícil, já que alterações sorológicas e radiológicas características muitas vezes estão ausentes, porém a termografia como método de diagnóstico precoce de doença articular infamatória apresentou sensibilidade, especificidade e precisão satisfatória na avaliação de doença articular inflamatória da articulação coxofemoral de cães (Jain, 1993).

A termografia pode ser um recurso auxiliar no diagnóstico de processos inflamatórios, demonstrada nos trabalhos de Roberto e Souza (2014), associados à medicina humana em Bandeira et al. (2012). Já na medicina veterinária, Purohit e Mccoy (1980) diagnosticaram a inflamação em cavalos de corrida por meio do emprego da termografia (Ring e Ammer, 2008).

As doenças geriátricas têm aparecido frequentemente nas clínicas, e os métodos semiológicos para fazer o diagnóstico provocam dor ao paciente dificultando o trabalho do médico veterinário e comprometendo o bem-estar dos animais. Além disso, as possibilidades terapêuticas para o tratamento dessas inflamações têm um custo alto, e um período de administração curto. $\mathrm{O}$ uso popular da semente de sucupira associada ao Biotônico Fontoura ${ }^{\circledR}$ tanto em humanos como em animais domésticos tem apresentado relatos bastante frequentes, levantando à hipótese deste trabalho, que teve como objetivo avaliar os efeitos dessa associação no tratamento da inflamação associada à inflamação da articulação coxofemoral no cão, empregando como diagnóstico um método próprio com radiação na faixa do infravermelho emitida pelo tecido lesado, registradas por um termógrafo.

\section{Material e Métodos}

O experimento realizou-se no Laboratório de Farmacologia do Departamento de Morfologia e Fisiologia Animal, ambos da Universidade Federal Rural de Pernambuco (UFRPE), para obtenção do extrato, e no Hospital Veterinário do Departamento de Medicina Veterinária da UFRPE, para avaliação clínica.

O Biotônico Fontoura ${ }^{\circledR}$, o Meloxicam, o Mesna, e as sementes de sucupira-branca foram adquiridos no comércio local da Região Metropolitana do Grande Recife, Pernambuco.

$\mathrm{O}$ extrato de sucupira-branca foi preparado de modo similar à utilização popular, acrescentando 136 gramas de semente de sucupirabranca, após procedimento de triagem e fragmentação, em $906 \mathrm{~mL}$ de Biotônico Fontoura ${ }^{\circledR}$, na proporção de $15 \%(\mathrm{~m} / \mathrm{v})$, ficando em infusão por sete dias, sob refrigeração a $4^{\circ} \mathrm{C}$. Depois desse período, o material foi filtrado, com filtro de $0,2 \mu \mathrm{m}$, sendo armazenado em frasco âmbar, contendo 52,5 mL de solução, suficiente para a administração diária na dose $300 \mathrm{mg}$ para cada animal conforme método estabelecido por Fukahori et al. (2015).

No Hospital Veterinário do Departamento de Medicina Veterinária da UFRPE, os animais eram submetidos a um protocolo de triagem que consistia no registro dos dados pessoais do tutor, características individuais do cão, avaliação clínica e ortopédica do paciente (Nogueira e Tudury, 2002), visando descartar alguma enfermidade concomitante com a lesão articular, que pudesse inviabilizar o animal para a pesquisa. Para enquadramento nos grupos de tratamento, os cães deveriam ser portadores de inflamação na 
articulação coxofemoral, exibindo sinais clínicos compatíveis com o quadro. Os tutores dos pacientes que se encaixaram nos pré-requisitos da pesquisa foram encaminhados para $o$ preenchimento das fichas cadastrais e de livre consentimento. Também foram informados sobre todas as etapas e exigências do projeto. Depois da assinatura dos tutores, os animais foram submetidos a exames radiográficos nas projeções laterolateral e ventrodorsal, para uma avaliação qualitativa das articulações e coleta de sangue para análise do hemograma, bioquímico e de alguns minerais que possam influenciar no processo de inflamação da articulação.

Depois das etapas de triagem clínica e avaliação radiográfica, todos os animais eram encaminhados para uma sala climatizada a $25^{\circ} \mathrm{C}$, umidade relativa do ar acima de $70 \%$, onde, após um período de 20 minutos, efetuou-se o registro da temperatura retal, mediante uso de termômetro clínico convencional; do mesmo modo, o registro da temperatura sobre o eixo da articulação coxofemoral em tomada lateral, mediante emprego de um termógrafo digital de captura de ondas eletromagnéticas na faixa espectral do infravermelho modelo TG165 da Flir Systems.

Em seguida, os animais foram divididos em dois grupos, GS (grupo sucupira-branca) e GM (grupo Meloxicam). No grupo GS, cada animal recebeu a orientação para administrar o extrato de sucupira-branca com Biotônico Fontoura ${ }^{\circledR}$ no volume de 2,5 mL, por via oral (v.o.), diariamente pela manhã, por 21 dias. No grupo GM, os animais recebiam (v.o.), diariamente pela manhã, cápsulas contendo Meloxicam na dose de $0,1 \mathrm{mg} / \mathrm{kg}$ por 21 dias, conforme orientação do fabricante. No início, $\mathrm{T} 0$, e no T10 dias, os animais do GM receberam 0,1 $\mathrm{mL}$ de Mesna $(100 \mathrm{mg} / \mathrm{mL})$.

O termógrafo tem uma resolução de 60 x 80 pixels, permitindo a obtenção de registro de temperatura em três segundos de exposição, com um foco de uma polegada de diâmetro se conservada uma distância de 24 polegadas da ponta do canhão do equipamento até a superfície aferida. A fim de minimizar as interferências externas sobre a leitura do equipamento, foi acoplada à sua saída uma cânula de PVC revestida internamente com película de tinta preta fosca com $30 \mathrm{~cm}$ de comprimento. No momento do registro das leituras de temperatura, a cânula e o equipamento eram justapostos sobre a região desejada, após posicionamento adequado do animal em cada registro. $\mathrm{O}$ foco, nessas condições, foi equivalente a uma área de $1,23 \mathrm{~cm}^{2}$, e a emissividade do equipamento foi ajustada para $\mathrm{e}=0,95 \mathrm{em}$ todas as aferições.

As leituras da temperatura termográfica $(\mathrm{Tt})$ e a temperatura retal $(\mathrm{Tc})$ foram realizadas em três momentos distintos, sempre entre 7 horas e 9 horas nos tempos iniciais T0, T10 dias e T21 dias após o início do tratamento, em ambiente climatizada a $25^{\circ} \mathrm{C}$, umidade relativa do ar acima de $70 \%$, por um período de 20 minutos. Com os dados obtidos ao final, foi providenciada a devida tabulação deles para, posteriormente, realizar os cálculos.

Determinou-se para cada animal de cada grupo de tratamento a diferença $\mathrm{Tc}-\mathrm{Tt}=\mathrm{DT}_{\text {cda }}$ dividindo o resultado por Tc, para normalizar os valores, ou seja, $\mathrm{DT}_{\text {cda }} / \mathrm{Tc}_{\mathrm{cda}}$, onde "cda" significa com doença articular. Para cada valor obtido, calculou-se o fator $\mathrm{FT}=\mathrm{DT}_{\text {cda }} / \mathrm{Tc}_{\mathrm{cda}}-0,19$; sendo 0,19 o valor médio referente ao parâmetro $\mathrm{DT}_{\text {sda }} / \mathrm{Tc}_{\text {sda }}$ obtido para os animais sem doença articular.

Se FT fosse zero ou negativo, os animais exibiriam temperaturas semelhantes aos animais sem doença articular; mas se FT fosse positivo, então a articulação exibiu termicamente alterações que puderam ser associadas à existência clínica de um processo inflamatório.

Os cálculos foram efetuados levando-se em consideração os registros de temperatura efetuados na dimensão lateral, sendo essa a posição que ofereceu resultados mais estáveis.

A análise estatística foi realizada mediante aplicação de um teste do tipo $\mathrm{t}$ de Student, disponível em planilha estatística do programa Excel versão 2010 da Microsoft sendo considerado como nível descritivo um valor de $\mathrm{p}<0,05$ (Reis, 2003).

\section{Resultados e Discussão}

Neste trabalho utilizou-se como método de diagnóstico a aplicação dos valores de temperatura aferidos com o termógrafo na faixa do infravermelho de animais portadores de inflamação na articulação coxofemoral, de acordo com a literatura disponível (Bandeira et al, 2012; Roberto e Souza, 2014; Segundo e Azevedo, 2015). Esse equipamento permitiu o registro de temperaturas em uma área de $1,23 \mathrm{~cm}^{2}$ sobre o eixo lateral da articulação, oferecendo um registro de temperatura local em $3 \mathrm{~s}$ de exposição.

A determinação do parâmetro FT fez-se necessária para que fosse possível avaliar exatamente a variação térmica entre a articulação e 
o ponto de referência térmica central do organismo, no caso a temperatura retal, sem a interferência das variações espontâneas que normalmente ocorrem sobre a articulação coxofemoral de cães sem doença inflamatória local.

$\mathrm{Na}$ Tabela 1, podem ser observados os valores do fator FT obtidos para cada cão sob tratamento com sucupira-branca, nos três momentos temporais considerados na pesquisa. Nesta tabela verifica-se a não ocorrência de diferença significativa entre os valores médios do fator FT obtidos nos períodos $\mathrm{T}=0$ e $\mathrm{T}=10$ dias; mas uma diferença importante apareceu entre FT obtido para $\mathrm{T}=21$ dias em relação ao período inicial $(\mathrm{T}=0)$, com valor de $\mathrm{p}=0,075$. Entretanto, um valor significativo foi obtido entre FT ( $\mathrm{T}=21$ dias) e FT ( $\mathrm{T}=10$ dias), para um valor de $\mathrm{p}<0,05$, marcando a evolução térmica dos animais avaliados em resposta ao tratamento com o preparado de extrato de sementes de sucupirabranca associado ao Biotônico Fontoura ${ }^{\circledR}$ na dose de $300 \mathrm{mg} / \mathrm{kg}$. Dessa forma, essa concentração foi importante no controle do processo inflamatório instalado. Basile et al. (2012) ressaltaram a ocorrência de limiares de detecção de processos inflamatórios nos diferentes sistemas musculoesqueléticos em equinos mediante uso da termografia.

Tabela 1. Variação do fator FT ao longo do tempo em cães portadores de inflamação articular tratados com sucupira-branca.

\begin{tabular}{cccccccccc}
\hline Dias & & $\mathbf{0}$ & & & $\mathbf{1 0}$ & & \multicolumn{2}{c}{$\mathbf{2 1}$} \\
\hline $\mathbf{A}$ & $\mathbf{T c}$ & $\mathbf{T t}$ & $\mathbf{F T}$ & $\mathbf{T c}$ & $\mathbf{T t}$ & $\mathbf{F T}$ & $\mathbf{T c}$ & $\mathbf{T t}$ & $\mathbf{F T}$ \\
\hline $\mathbf{1}$ & 38,5 & 34,7 & $-0,091$ & 38,6 & 27,6 & 0,095 & 38,2 & 28,8 & 0,056 \\
$\mathbf{2}$ & 38,0 & 30,6 & 0,005 & 38,8 & 31,3 & 0,003 & 38,1 & 29,2 & 0,043 \\
$\mathbf{3}$ & 38,3 & 33,0 & $-0,052$ & 38,2 & 30,0 & 0,025 & 38,2 & 29,1 & 0,048 \\
$\mathbf{4}$ & 38,1 & 27,2 & 0,096 & 38,6 & 25,7 & 0,144 & 39,1 & 25,6 & 0,155 \\
$\mathbf{5}$ & 39,3 & 26,8 & 0,128 & 39,2 & 30,0 & 0,045 & 38,7 & 27,4 & 0,102 \\
$\mathbf{6}$ & 38,9 & 28,4 & 0,080 & 38,9 & 32,0 & $-0,013$ & 38,2 & 31,1 & $-0,004$ \\
$\mathbf{7}$ & 38,2 & 33,0 & $-0,054$ & 38,5 & 32,0 & $-0,021$ & 38,8 & 30,4 & 0,026 \\
$\mathbf{8}$ & 38,7 & 28,1 & 0,094 & 39,0 & 31,7 & $-0,003$ & 39,0 & 26,2 & 0,138 \\
$\mathbf{9}$ & 38,4 & 30,4 & 0,018 & 38,6 & 33,0 & $-0,045$ & 38,9 & 25,9 & 0,144 \\
$\mathbf{1 0}$ & 39,2 & 28,0 & 0,096 & 39,2 & 28,0 & 0,096 & 39,0 & 27,5 & 0,295 \\
$\mathbf{1 1}$ & 39,1 & 27,9 & 0,096 & 38,9 & 27,8 & 0,095 & 38,7 & 25,9 & 0,141 \\
$\mathbf{1 2}$ & 37,9 & 27,1 & 0,095 & 38,3 & 27,0 & 0,105 & 39,2 & 26,0 & 0,145 \\
$\mathbf{1 3}$ & 39,2 & 26,8 & 0,126 & 38,4 & 26,4 & 0,123 & 38,0 & 25,8 & 0,131 \\
$\mathbf{1 4}$ & 39,0 & 28,8 & 0,072 & - & - & - & - & - & - \\
$\mathbf{1 5}$ & 38,5 & 25,8 & 0,140 & - & - & - & - & - & - \\
\hline Média & & & 0,056 & & & 0,050 & & & 0,095 \\
Desvio & & & 0,072 & & & 0,063 & & 0,056 \\
P0 & & & - & & & 0,409 & & - & 0,075 \\
P10 & & & - & & & - & & 0,039 \\
\hline
\end{tabular}

Notas: $1 \mathrm{Tc}$ - Temperatura do corpo " ${ }^{\circ} \mathrm{C} " ; \mathrm{Tt}$ - Temperatura registrada no termógrafo "o $\mathrm{C} " ; \mathrm{FT}-$ Fator temperatura.

2 "po" - probabilidade em relação ao tempo zero; "p10" - probabilidade em relação aos valores do tempo de 10 dias.

Na Tabela 2, verificam-se os valores do fator FT obtidos para animais clinicamente incluídos na condição de inflamação na articulação coxofemoral tratados por período similar com Oxicam. Nesta tabela, acompanhando os valores de probabilidade, verificou-se a não ocorrência de diferença significativa entre os valores médios de FT obtidos nos três intervalos temporais, para um valor de $\mathrm{p}<0,05$. Esse resultado sugeriu que o Meloxicam, na dose de $0,1 \mathrm{mg} / \mathrm{kg}$, parece não ter contribuído para as modificações térmicas observadas na articulação coxofemoral, denunciando baixo impacto sobre o processo inflamatório situado na região articular.
O Biotônico Fontoura ${ }^{\circledR}$ é um tônico formado pela associação de diferentes princípios ativos tais como sulfato ferroso $(0,833 \mathrm{mg})$, ácido fosfórico $(4,666 \mathrm{mg})$ em excipientes q.s.p. 1,0 mL. Nesse excipiente podem ser encontrados extrato glicólico (Aloe perryi, Commiphora myrrha, Myristica fragan, Cinnamomum zeylanicum, metilparabeno, propilparabeno e propilenoglicol), sacarose, sorbasto de potássio, corante caramelo, aroma e água de osmose reversa. Essa formulação proposta pela indústria Hypermarcas S.A. tem como propósito oferecer um suplemento mineral apropriado para corrigir anemias carenciais principalmente quando o organismo do animal é 
submetido a formulação de dietas inadequadas. Acreditamos, portanto, na possibilidade de existência de sinergismo entre esses componentes dessa formulação e nas substâncias presentes no extrato bruto de sucupira-branca.

A associação da sucupira-branca com o Biotônico Fontoura ${ }^{\circledR}$ ofereceu um efeito de transformação do processo inflamatório mais impactante do que aquele mediado pelo Meloxicam, obtendo-se as expressões matemáticas que descreveram a variação do Fator FT em cada condição de tratamento. Ao longo dos intervalos temporais considerados, obtendo-se equações do tipo polinomiais, pôde-se descobrir a taxa de variação infinitesimal desses fatores em cada condição de tratamento, obtendo-se a derivada primeira ambas as funções; ou seja FTM' = $0,0002 \mathrm{t}+0,018$ para os animais tratados com Meloxicam e FTS' $=0,0018-0,02$ para os animais tratados com sucupira-branca.

As derivadas são funções temporais, o que significa que para cada momento a produção térmica local depende do tempo de avaliação. Escolhendo-se, portanto, o tempo de 21 dias para se verificar a diferença entre as taxas de variação de temperatura, encontramos a razão TFS'/TFM' $=$ 1,61 que significou que a taxa de variação térmica local nos animais tratados com sucupira-branca foi $61 \%$ maior do que a taxa de variação térmica observada para os animais tratados com Meloxicam.

As derivadas são funções temporais, o que significa que para cada momento a produção térmica local depende do tempo de avaliação. Escolhendo-se, portanto, o tempo de 21 dias para se verificar a diferença entre as taxas de variação de temperatura, encontramos a razão TFS' $/$ TFM' $^{\prime}=$ 1,61 que significou que a taxa de variação térmica local nos animais tratados com sucupira-branca foi $61 \%$ maior do que a taxa de variação térmica observada para os animais tratados com Meloxicam.

Tabela 2. Variação do fator FT ao longo do tempo em cães portadores de inflamação articular tratados com Meloxicam.

\begin{tabular}{|c|c|c|c|c|c|c|c|c|c|}
\hline Dias & & 0 & & & 10 & & & 21 & \\
\hline A & Tc & $\mathbf{T t}$ & FT & Te & $\mathbf{T t}$ & FT & Tc & $\mathbf{T t}$ & FT \\
\hline 1 & 39,2 & 23,5 & 0,211 & 38,9 & 27,5 & 0,103 & 39,2 & 26,6 & 0,131 \\
\hline 2 & 38,8 & 29,2 & 0,057 & 39,1 & 31,2 & 0,012 & 39,3 & 31,1 & 0,019 \\
\hline 3 & 39,0 & 29,6 & 0,51 & 39,1 & 27,6 & 0,104 & 39,3 & 33,7 & $-0,048$ \\
\hline 4 & 38,3 & 27,5 & 0,092 & 38,4 & 28,0 & 0,081 & 38,5 & 27,7 & 0,091 \\
\hline 5 & 38,4 & 24,7 & 0,167 & 38,3 & 26,6 & 0,116 & 38,6 & 31,7 & $-0,011$ \\
\hline 6 & 38,4 & 25,6 & 0,143 & 38,3 & 24,4 & 0,173 & 38,4 & 25,4 & 0,149 \\
\hline 7 & 36,8 & 30,6 & 0,017 & 38,8 & 27,8 & 0,094 & 38,9 & 21,9 & 0,247 \\
\hline 8 & 37,8 & 30,2 & 0,011 & 38,8 & 27,3 & 0,104 & 37,6 & 21,8 & 0,230 \\
\hline Média & & & 0,094 & & & 0,190 & & & 0,101 \\
\hline Desvio & & & 0,007 & & & 0,254 & & & 0,109 \\
\hline $\mathbf{P}_{0}$ & & & - & & & 0,161 & & & 0,439 \\
\hline $\mathbf{P}_{10}$ & & & - & & & - & & & 0,190 \\
\hline
\end{tabular}

O fator FT para cada animal do grupo tratado com extrato de sucupira-branca associado ao Biotônico Fontoura ${ }^{\circledR}$ mudou ao longo do tempo, o que, de certa forma, assinalou a evolução do processo inflamatório em todos os animais envolvidos. Observa-se o deslocamento dos fatores FT conforme o avanço do tratamento até 21 dias, o que está de acordo com as transformações do processo inflamatório, à medida que o cenário bioquímico que permeia a matriz extracelular e o meio interno citoplasmático sofrem alterações importantes. Alterações que visam à diminuição dos processos pró-inflamatórios e à potencialização de ações anti-inflamatórias. Desse modo, culminando com o acionamento de mecanismos moleculares que visam à obtenção do reequilíbrio do metabolismo local, assim como o reparo dos processos lesivos, acima de tudo, com a restauração da qualidade do fluxo circulatório local (Wannamacher e Ferreira, 2006; Tasaka, 2011).

$\mathrm{O}$ fator FT pode ser tanto positivo quanto negativo. Se for positivo, o valor do fator FT sugere a presença de um processo inflamatório instalado sobre os tecidos que envolvem a articulação coxofemoral em cães. Quanto mais próximo de zero, menor a diferença $(\mathrm{Tc}-\mathrm{Tf}) / \mathrm{Tc}$ o que sugere uma temperatura elevada na articulação, em relação à temperatura corpórea referencial. Quando o fator FT for zero, significa que seu valor é exatamente igual ao valor médio de FT obtido para 
animais sem doença articular nem inflamação. Quando o FT for negativo, significa que a variação térmica na articulação em relação à temperatura corpórea de referência é maior em relação aos animais não portadores de doença articular, sendo essa diferença maior quanto mais próximo de zero for o valor do Fator FT.

$\mathrm{Na}$ avaliação bioquímica dos animais tratados com extrato de sucupira-branca e o Biotônico Fontoura ${ }^{\circledR}$, mediante amostras de sangue coletados no início $(\mathrm{T}=0)$ e no fim do tratamento ( $\mathrm{T}=21$ dias), não se observaram diferenças significativas perante os parâmetros avaliados, tendo como base de referência, os parâmetros demonstrados por Kaneko et al. (1997). Porém, na análise dos parâmetros relacionados com o ácido úrico, observou-se que os cães apresentavam um aumento importante em relação aos valores padrões e, após 21 dias de tratamento com extrato de sucupira-branca mais o Biotônico Fontoura ${ }^{\circledR}$, os níveis de ácido úrico diminuíram em 25,6\%, aproximando-se do limite padrão.

$\mathrm{O}$ ácido úrico é uma substância produzida naturalmente pelo organismo como o produto final da degradação metabólica de ribonucleotídeos purínicos endógenos e de ácidos nucléicos, principalmente oriundo dos alimentos. Na maior parte dos animais, o ácido úrico é transformado em alantoína por meio do sistema enzimático das uricases, normalmente presente no tecido hepático, sendo mais solúvel e facilmente eliminada pelo sistema renal, dependendo das condições metabólicas locais (Colville, 2010). É importante assinalar que deficiências na produção das uricases, como ocorre com certa facilidade em cães da raça dálmata, pode ocorrer o acúmulo de ácido úrico gerando problemas, por exemplo, a formação de cristais em alguns tecidos, a exemplo do próprio tecido renal, e as consequências advindas, como intensa reação inflamatória.

O extrato de sucupira-branca favoreceu a diminuição da concentração de ácido úrico em 21 dias de tratamento. Embora não exista comprovação de ser possível que pelo menos parte dos processos inflamatórios sobre as estruturas da articulação coxofemoral pode ser decorrente da deposição de cristais, principalmente na forma de uratos de sódio nas articulações, potencializando o desenvolvimento de processo inflamatório do tipo artrite.

Quanto aos parâmetros relacionados com o eritograma de animais com inflamação na articulação coxofemoral, tratados com sucupira- branca, não se encontraram diferenças significativas para um valor descritivo de $\mathrm{p}<0,05$ tendo como base de referência os parâmetros demonstrados por Jain (1993).

O método empregado para a obtenção do fator FT revelou $80 \%$ de sensibilidade, ou seja, há capacidade de se encontrar animais clinicamente enquadrados na condição de inflamação na articulação coxofemoral, apresentando o fator FT positivo. Contudo, nada impede que animais com FT negativo, em condições especiais, apresentem sinais clínicos da inflamação. Isso ocorre porque um processo inflamatório é um evento multifatorial, complexo e dinâmico; pode assumir particularidades no momento em que as sinalizações moleculares internas sejam compatibilizadas com as programações metabólicas estabelecidas pelos respectivos tecidos; assim como a qualidade da coordenação das principais cascatas de eventos que culminam no restabelecimento de um novo referencial metabólico, para que as atividades fisiológicas sejam retomadas, garantindo um grau satisfatório de atividade orgânica compatível com a manutenção das condições mínimas de bem-estar animal.

O processo inflamatório é um evento característico, multifatorial, complexo e dinâmico, podendo assumir características particulares na medida em que as sinalizações moleculares internas sejam compatibilizadas com as programações metabólicas estabelecidas pelos respectivos tecidos; igualmente, a qualidade da coordenação das principais cascatas de eventos que culminam no restabelecimento de um novo referencial metabólico, para que as atividades fisiológicas sejam retomadas, garantindo um nível satisfatório de atividade orgânica compatível com a manutenção das condições mínimas de bem-estar animal.

Outro aspecto é que não foram encontradas diferenças significativas entre os valores dos fatores FT em relação a machos e fêmeas com $\mathrm{p}=$ 0,648 para uma avaliação da articulação em uma perspectiva lateral, tanto entre animais tratados com sucupira-branca como em animais tratados com Meloxicam.

Também é importante mencionar que não foram encontradas diferenças significativas nas radiografias de animais tratados com sucupirabranca em associação com o Biotônico Fontoura ${ }^{\circledR}$ ao longo do período de tratamento de 21 dias, o que está de acordo com Purohit et al. (2003), que 
assinalam a dificuldade de registro do processo inflamatório mediante emprego de técnicas radiográficas.

A termografia possibilitou a verificação de diferenças térmicas importantes que foram associadas às transformações locais do metabolismo em resposta a estímulos próinflamatórios, como também à ação de substâncias oriundas de extratos vegetais, como é o caso da sucupira.

\section{Conclusão}

As preparações que associam o extrato de sementes de sucupira-branca com o Biotônico Fontoura ${ }^{\circledR}$ conseguiram reduzir o processo inflamatório sobre a articulação coxofemoral em cães na dose de $300 \mathrm{mg} / \mathrm{kg}$, constatada pela anamnese associada à captura da temperatura local por meio do aparelho termográfico.

As transformações térmicas avaliadas pela termografia permitem observar a involução do processo inflamatório de cães tratados com o extrato de sementes de sucupira-branca com o Biotônico Fontoura ${ }^{\circledR}$ e de cães tratados com Meloxicam.

Os cães do presente estudo se encontravam em um momento inicial da doença inflamatória, sem apresentar alterações nos exames bioquímico, hemograma e radiográfico, porém apresentavam dor e outros sinais clínicos compatíveis com o quadro de inflamação. A temperatura capturada pelo aparelho termográfico se enquadrava nos parâmetros padronizados referentes a processos inflamatórios.

Apesar de não ter sido encontradas informações a respeito do envolvimento do ácido úrico nas doenças inflamatórias na medicina veterinária, a elevação dos parâmetros do ácido úrico dos animais desta pesquisa no exame inicial e sua regressão à normalidade depois do uso do extrato, levanta a hipótese de que, em alguns casos, os animais também podem sofrer a influência do ácido úrico em casos de inflamação da articulação.

São necessários novos experimentos para aprimorar o método, aumentando a sua sensibilidade, assim como o descarte ou inclusão do ácido úrico no mecanismo do processo inflamatório das articulações de cães, e ainda determinar que este seria o alvo do extrato da semente de sucupira para a remissão dos sintomas e do quadro clínico dos processos inflamatórios articulares na medicina veterinária.

\section{Conflito de Interesse}

Os autores declaram não existir conflito de interesse.

\section{Comitê de Ética}

A pesquisa recebeu a aprovação e o parecer da Comissão de Ética para Experimentação Animal da Universidade Federal Rural de Pernambuco, sob o de número de Protocolo 063/2016.

\section{Referências}

Bandeira, F.; Moura, M.A.M.; Souza, M.A.; Nohama, P.; Neves, E.B. Pode a termografia auxiliar no diagnóstico de lesões musculares em atletas de futebol? Revista Brasileira Medicina Esporte, 18(4): 246-251, 2012.

Basile, R.C.; Filho, D.Z.; Polo, T.S.; Queiroz-Neto, A.; Macoris, D.G. Acompanhamento póscirúrgico de desmotomia do ligamento anular plantar de equino com auxílio de termografia quantitativa: relato de caso. Revista Veterinária Zootecnia Minas, 109: 131-132, 2011.

Colville, T.P. Anatomia e Fisiologia Clínica para Medicina Veterinária. In: Colville, T.P.; Bassert, J.M. Anatomia e fisiologia clínica para medicina veterinária. Rio de Janeiro: Elsevier, 2010.

Fukahori, F.L.P.; Rêgo, M.S.A.; Lira, F.J.C.; Dias, M.B.M.C.; Leitão, R.S.C.; Silva, V.C.L.; Silva Neto, J.F.; Souza, D.M.B.; Gimenez, G.C.; Lima, E.R. Atividade espontânea e determinação da toxicidade do óleo essencial da semente de sucupira (Pterodon emarginatus, Vogel) usado em camundongos. Ciência Veterinária nos Trópicos, 18(3): 18-29, 2015.

Jain, N. C. (ed). Essentials of veterinary hematology. Philadelphia: Lea and Febiger, 1993. 420p.

Kaneko, J.J.; Harvey, J.W.; Bruss, M.L. (ed.). Clinical biochemistry of domestic animals. $5^{\text {th }}$ ed. New York: Academic Press, 1997. 932p.

Lorenzi, H.; Matos, F.J.A. Plantas medicinais do Brasil: Nativas e exóticas. Nova Odessa: Instituto Plantarum, 2002. 576p.

Moraes, W.F.; Galdino, P.M.; Nascimento, M.V.M.; Vandeerlinde, F.A.; Bara, M.T., Costa, E.A., de Paula, J.R. Triterpenoids involved in the anti-inflamatory effect of ethanolic extract of Pterodon emarginatus 
Vogel stem bark. Journal of Natural Medicines, 66: 202-207, 2012.

Nogueira, S.R.; Tudury, E.A. Exame clínico ortopédico em cães e gatos: part 1. Clínica Veterinária, 36: 34-58, 2002.

Nogueira, S.R.; Rocha, L.B.; Tudury, E.A. Utilização do índice de distração no diagnóstico da displasia coxofemoral canina. Clínica Veterinária, 54: 28-42, 2005.

O'Reilly, J.D.; Mallet, A.J.; Mcanlis, G.T.; Young, I.S.; Halliwell, B. Consumption of flavonoids in onions and black tea lack of effect on flavonoids from Stachys chrysantha and Stachys candida. Biological and Pharmaceutical Bulletin, 23(1): 47-53, 2000.

Purohit, R.C.; Mccoy, M.D. Thermography in the diagnosis of inflamatory process in the horse. American Journal of Veterinary Research, 41(8): 1167-1174, 1980.

Purohit, R.C.; Schumacher, J.; Molloy, J.M.; Smith, J.W.; Pascoe, D.D. Elucidation of thoracic and lumbosacral dermatomal patterns in the horse. International Journal of Thermal Sciences, 13: 79, 2003.

Reis, J.C. Comparação de grupos. In:

Estatística aplicada à pesquisa em ciência veterinária. Olinda: Luci Artes Gráficas, 2003. p.313-320.

Ring, E.F.J.; AMMER, K. Thermal imaging in the disease of the skeletal and neuromuscular systems. In: Diakides, N.A.; Bronzino, J.D. Medical infrared imaging, 17: 1-15, 2008.

Roberto, J.V.B.; Souza, B.B. Utilização da termografia de infravermelho na medicina veterinária e na produção animal. Journal of Animal Behaviour and Biometeorology, 2(3): 73-84, 2014.

Rocha, L.B.; Tudury, E.A.; Roehsig, C.; Baraúna, D.; Chioratto, R.; Araújo, F.P.; Kemper, B. Denervação articular coxofemoral em cães com doença articular degenerativa secundária à displasia. Ciência Animal Brasileira, 14(1): 120-134, 2013.

Segundo, F.A.S.; Azevedo, A.S. Termografia na avaliação de processo inflamatório. Congresso Brasileiro de Medicina Veterinária e Congresso Sul-Brasileiro da ANCLIVEPA, 1. 2015, Curitiba. Anais [...]. Curitiba, 2015. p. 0278-0282.

Spindola, H.M.; Servat, L.; Denny, C.; Rodrigues, R.A.F.; Eberlin, M.N.; Cabral, E.; Sousa, I.M.; Tamashiro, J.Y.; Cravalho, J.E.; Foglio, M.A. Antinociceptive effect of geranylgeraniol and 6 $\alpha, 7 \beta-$ dihydroxyvouacapan-17ß-oate methyl ester isolated from Pterodon pubescens Benth. BMC. Pharmacology and Toxicology, 10: 110, 2010.

Tasaka, A.C. Anti-inflamatórios não esteroidais. In: Spinosa, H.S.; Górniak, S.L.; Bernardi, M.M. Farmacologia aplicada à medicina veterinária de Spinosa. $5^{\text {a }}$ ed., Rio de Janeiro: Guanabara Koogan, 2011.p.245-260.

Wannamacher, L.; Ferreira, M.B.C. Antiinflamatórios não-esteroides. In: Fuchs, F.D.; Wannamacher, L.; Ferreira, M.B.C. Farmacologia clínica: fundamentos da terapêutica racional. $3^{\mathrm{a}}$ ed. Rio de Janeiro: Guanabara Koogan, 2006. p.298-305. 